\title{
EDITORIAL
}

\section{Genetics and epigenetics as tools to inform the pathophysiology of neuropsychiatric disorders}

\author{
Gabriel Rodrigo Fries iD \\ Translational Psychiatry Program, Department of Psychiatry and Behavioral Sciences, The University of Texas Health Science Center at \\ Houston (UTHealth), Houston, TX, USA. iD https://orcid.org/0000-0002-5468-2612
}

Most neuropsychiatric disorders are regarded as multifactorial conditions involving a complex interplay between genetic variants and the environment. In general, this suggests that genetic variants that may confer risk to a disorder typically require a specific environmental context that will influence its expression and activity. This concept has also been used to suggest the potential role of epigenetic mechanisms in conferring risk to these disorders, due to their ability to change in response to environmental stimuli. Regardless of their overall penetrance in a given diagnosis, identifying genetic and epigenetic targets can have a significant impact not only on the development of novel treatments, but also provide informative and clinically useful biomarkers for a plethora of conditions.

There are several different approaches to studying the genetics of complex diseases such as neuropsychiatric disorders. The obvious and most traditional one involves the cross-sectional comparison between patients and matched controls, which is expected to provide disease biomarkers. For instance, a recent study published in the Brazilian Journal of Psychiatry compared 462 patients with schizophrenia and 598 healthy controls and identified a significant association between the disorder and the frequency of a genetic variant within the $5^{\prime}$-nucleotidase II (NT5C2) gene. ${ }^{1}$ This approach has been used often since its advent in early studies of genetics, and has provided at least two important cues for the study of genetics of neuropsychiatric disorders: 1 ) most such disorders are associated with multiple genetic variants, each having a small effect; and 2) very large sample sizes are needed for successful identification of these variants. This acquired knowledge has shaped the field over the years and fostered the formation of consortia where several independent groups combine the results of their own populations into one large analysis with reasonable statistical power. A successful example of this approach is the Psychiatric Genetics Consortium (PGC, http://www.med. unc.edu/pgc), which has been leading the discovery of genetic variants implicated in severe mental illness, such as schizophrenia, bipolar disorder, and major depression. ${ }^{2}$

Another approach to the study of genetics and epigenetics is the investigation and discussion of genetic variants as 'modifiers' rather than determinants of disease.

Correspondence: 1941 East Rd, 77054, Houston, TX, USA.

E-mail: Gabriel.R.Fries@uth.tmc.edu
Examples of this approach include studying the effects of specific markers on clinical symptoms, age at illness onset, differential prognosis, responsiveness to treatment, and other clinical variables. A recent study published in this journal, for instance, has identified genetic determinants of the age at onset of Alzheimer's disease, ${ }^{3}$ and similar findings have been reported for several other disorders. In addition, several studies have acknowledged a key role of genetic and epigenetic alterations in neuroanatomical and neurocognitive alterations. A genetic variant within the catechol-O-methyltransferase (COMT) gene has been recently associated with cognitive measures in patients with schizophrenia, ${ }^{4}$ and a specific variant (Val66Met) of the brain-derived neurotrophic factor gene (BDNF) has been linked to memory deficits in older subjects. ${ }^{5}$ These findings suggest that genetic markers can inform specific prognosis and define patient subgroups (for instance, those who may need special neurocognitive-focused therapies), among other implications.

All in all, while it is clear that genetic and epigenetic studies have much to contribute to the field of neuropsychiatry, clinical use of such discoveries is still quite limited. This is expected to change as the genetic underpinnings of neuropsychiatric disorders become increasingly understood. For this to occur, genome-wide approaches, as opposed to traditional candidate gene studies, are slowly becoming the norm rather than the exception, and we are starting to see a gradual shift from microarray-based to next-generation sequencing investigations. Although both of these changes require massive sample sizes and are much more costly than (limited) conventional methods, they are expected to significantly improve the identification of common and rare variants in these disorders in the years to come. The same trend is being observed in epigenomic studies, with accurate detection of pathologically relevant methylation markers. Finally, the development of sophisticated statistical genetics and bioinformatics tools that allow the integration of multiple 'omics' (such as phenomic, genomic, methylomic, and transcriptomic alterations) into one single analysis should significantly improve our ability to detect highly specific and clinically relevant hits, thereby allowing genetic findings to become accessible to patients and physicians.

How to cite this article: Fries GR. Genetics and epigenetics as tools to inform the pathophysiology of neuropsychiatric disorders. Braz J Psychiatry. 2019;41:5-6. http://dx.doi.org/10.1590/15164446-2018-0329 


\section{Disclosure}

The author reports no conflicts of interest.

\section{References}

1 Li Z, Jiang J, Long J, Ling W, Huang G, Guo X, et al. The rs11191580 variant of the NT5C2 gene is associated with schizophrenia and symptom severity in a South Chinese Han population: evidence from GWAS. Braz J Psychiatry. 2017;39:104-9.

2 Sullivan PF, Agrawal A, Bulik CM, Andreassen OA, Borglum AD, Breen G, et al. Psychiatric genomics: an update and an agenda. Am J Psychiatry. 2018;175:15-27.
3 Oliveira FF, Chen ES, Smith MC, Bertolucci PH. Associations of cerebrovascular metabolism genotypes with neuropsychiatric symptoms and age at onset of Alzheimer's disease dementia. Braz J Psychiatry. 2017;39:95-103.

4 Matsuzaka CT, Christofolini D, Ota VK, Gadelha A, Berberian AA, Noto $\mathrm{C}$, et al. Catechol-O-methyltransferase (COMT) polymorphisms modulate working memory in individuals with schizophrenia and healthy controls. Braz J Psychiatry. 2017;39:302-8.

5 Azeredo LA, De Nardi T, Levandowski ML, Tractenberg SG, Kommers-Molina J, Wieck $A$, et al. The brain-derived neurotrophic factor (BDNF) gene Val66Met polymorphism affects memory performance in older adults. Braz J Psychiatry. 2017;39:90-4. 